\title{
Results from the OPERA experiment in the CNGS beam
}

\section{Gabriele Sirri*†}

Istituto Nazionale di Fisica Nucleare, Sez. di Bologna

E-mail: gabriele.sirri@bo.infn.it

The OPERA experiment at the Gran Sasso underground laboratory has been designed to study the $v_{\mu} \rightarrow v_{\tau}$ oscillation in appearance mode in the CNGS neutrino beam. Five $v_{\tau}$ candidate events have been recently confirmed, using an extended sub-sample of data from the 2008-2012 runs. Given the number of analysed events and the low background, $v_{\mu} \rightarrow v_{\tau}$ oscillations have been established with a significance of $5 \sigma$. We report results based on an increased sample of scanned emulsion target units (bricks). The $v_{\tau}$ data analysis will be updated and discussed. The analysis of the collected electron neutrino sample and the analysis of the muon charge ratio in the cosmic ray sample will also be covered.

The European Physical Society Conference on High Energy Physics 22-29 July 2015

Vienna, Austria

* Speaker.

${ }^{\dagger}$ on behalf of the OPERA Collaboration 


\section{Introduction}

The OPERA (Oscillation Project with Emulsion tRacking Apparatus) experiment [1, 2] was designed to conclusively prove the existence of $v_{\mu} \rightarrow v_{\tau}$ oscillations in an almost pure $v_{\mu}$ beam. The direct appearance search is based on the detection of $\tau$ leptons produced in $v_{\tau}$ charged current interactions (CC). The OPERA detector was placed in the underground Gran Sasso Laboratory (LNGS), $730 \mathrm{~km}$ away from the neutrino source, in the high energy long-baseline CERN to LNGS beam (CNGS) [3, 4]. The average neutrino energy is about $17 \mathrm{GeV}$. The $\bar{v}_{\mu}$ contamination is $2.1 \%$ in terms of interactions; the $v_{e}$ and $\bar{v}_{e}$ contaminations are in total below $1 \%$, while the intrinsic $v_{\tau}$ component (from $D_{s}$ decays in the CNGS target and beam-dump) is of $\mathrm{O}\left(10^{6}\right)$, hence negligible.

\section{The OPERA detector}

The flight length of $\tau$ leptons for the CNGS beam has a roughly exponential distribution with a mean value of about $600 \mu \mathrm{m}$. The OPERA detector is designed to achieve a micrometric tracking accuracy over a very large detector volume of $(6.5 \times 6.5 \times 8) \mathrm{m}^{3}$.

The target is a highly modular structure of nuclear emulsion based trackers (bricks). A brick is composed of 57 emulsion films interleaved with $56,1 \mathrm{~mm}$ thick, lead plates for a mass of $8.3 \mathrm{~kg}$. Its thickness along the beam direction corresponds to about 10 radiation lengths and its transverse size is $128 \times 102 \mathrm{~mm}^{2}$. A nuclear emulsion film consists of two $44 \mu \mathrm{m}$ layers deposited on each side of a $205 \mu \mathrm{m}$ plastic base. Bricks are arranged in 28 vertical planes (walls) interspersed with pairs of planes of horizontal and vertical scintillator strips (Target Tracker or TT) allowing to locate the target unit in which the neutrino interaction occurred with an $\mathrm{O}(\mathrm{cm})$ resolution.

The detector (Fig. 1) is divided into two identical units called Super Modules (SM), each consisting of a target and a magnetic spectrometer instrumented with Resistive Plate Chamber (RPC) detectors and high-precision Drift Tubes (DT). The average number of bricks has been about 140000 for a target mass of about $1.2 \mathrm{kt}$.

The electronic detectors provide timing for beam synchronization, define 3D probability density maps for neutrino interaction vertex position, identify muons and measure their charge and momentum. Decay and interaction topologies are reconstructed from nuclear emulsion data obtained by automated optical microscopes with $\mathrm{O}(\mu \mathrm{m})$ resolution.

\section{Data analysis and event selection}

The OPERA detector has been exposed to the CNGS beam $v_{\mu}$ beam from 2008 to 2012. A total exposure corresponding to $17.97 \times 10^{19}$ protons on target (p.o.t.) resulted in 19505 neutrino interactions in the target fiducial volume.

Events are classified as charged current-like ( $1 \mu$-like) if a track has a path length larger than $660 \mathrm{~g} / \mathrm{cm}^{2}$ or the total number of TT and RPC planes having at least one hit is larger than 19; the complementary sample is defined as neutral-like ( $0 \mu$-like) [5].

Bricks are ranked according to their (decreasing) probability to contain the neutrino interaction vertex. The most probable brick ( $1^{\text {st }}$ brick) is extracted from the wall asynchronously with respect to the beam exposure to allow for film development, scanning and for the search for $\tau$ decays. If 


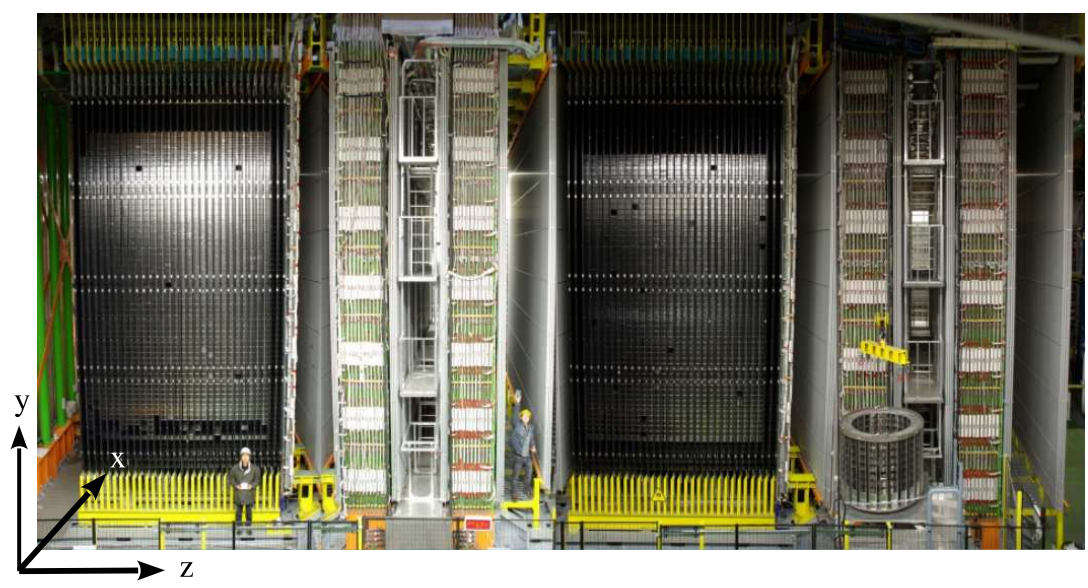

Figure 1: A picture of the OPERA detector. CNGS neutrinos travel from left to right. The (right-handed) reference frame is oriented such that: the $y$-axis is perpendicular to the hall floor and pointing up; the $z$-axis is orthogonal to the brick walls and oriented as the incoming neutrinos. The angle between the neutrino direction and the $z$-axis projected into the $y z$ plane is of $58 \mathrm{mrad}$.

the neutrino interaction vertex is not found in this brick, it is searched for in the next brick in the probability ranking ( $2^{\text {nd }}$ brick).

The vertex location procedure in one brick starts from a set of predictions provided by the electronic detectors; then, tracks of secondary particles produced in a neutrino interaction are followed back in the brick, film by film, from the most downstream one to the interaction point where they originate. Whenever a track disappearance signal is detected (the track is not found in three consecutive films), a volume of $1 \mathrm{~cm}^{2}$ for 5 films upstream and 10 films downstream of the candidate vertex point is scanned in order to fully reconstruct the event and find any decay candidate through a dedicated decay search procedure.

If secondary vertices or decays are found, a full kinematical analysis is performed combining the measurements in the nuclear emulsion with data from the electronic detectors. The momentum of charged particles can be measured in emulsions by the Multiple Coulomb Scattering up to $6 \mathrm{GeV} / \mathrm{c}$ with a resolution better than $22 \%$ using the angular deviations [6]. It can be measured up to $12 \mathrm{GeV} / \mathrm{c}$ with a resolution better than $33 \%$ using position deviations. For muons crossing the spectrometers, the momentum is measured with a resolution better than $22 \%$ up to $30 \mathrm{GeV} / \mathrm{c}$; the muon charge is also determined [5]. The hint of a decay topology is the observation of an impact parameter larger than $10 \mu \mathrm{m}$, defined as the minimum distance between the track and the reconstructed vertex, excluding low momentum tracks. The appearance of the $\tau$ lepton is identified by the detection of its characteristic decay topologies, either in one prong (electron, muon or hadron) or in three prongs.

Kinematical selection criteria are then applied according to the decay channel.

\section{Result of the $v_{\tau}$ search}

The sample of events analyzed so far consists of the $1^{\text {st }}$ and $2^{\text {nd }}$ bricks, for all $0 \mu$-like events and for $1 \mu$-like events with a muon momentum $p_{\mu}<15 \mathrm{GeV} / \mathrm{c}$. The number of events with 
a completed decay-search for the present analysis amounts to 5408 (Tab. 1). Five candidates $\tau$ events have been observed, satisfying the kinematic selection criteria.

\begin{tabular}{lcccccc}
\hline \hline & 2008 & 2009 & 2010 & 2011 & 2012 & Total \\
\hline p.o.t. $\left(10^{19}\right)$ & 1.74 & 3.53 & 4.09 & 4.75 & 3.86 & 17.97 \\
$0 \mu$ events & 149 & 253 & 268 & 270 & 204 & 1144 \\
$1 \mu$ events $\left(p_{\mu}<15 \mathrm{GeV} / \mathrm{c}\right)$ & 542 & 1020 & 968 & 966 & 768 & 4264 \\
Total events & 691 & 1273 & 1236 & 1236 & 972 & 5408 \\
Detected $v_{\tau}$ candidates & - & 1 & - & 1 & 3 & 5 \\
\hline \hline
\end{tabular}

Table 1: Number of events used in this analysis and number of the detected $v_{\tau}$ candidates for each run year.

The first $v_{\tau}$ candidate (Fig. 2, top left) was observed in the 2008-2009 data sample and described in detail in [7]. It consists of a 7-prongs neutrino interaction. One of the tracks exhibits a kink topology and the daughter track is identified as a hadron through its interaction. Two $\gamma$ rays points to the secondary vertex. Their invariant mass $\left((120 \pm 20(\right.$ stat. $) \pm 35($ syst. $\left.)) \mathrm{MeV} / \mathrm{c}^{2}\right)$ is compatible with the $\pi^{0}$ mass; their combination with the secondary hadron, assumed to be a $\pi^{-}$, gives an invariant mass of $640_{-80}^{+125}(\text { stat. })_{-90}^{+100}($ syst. $) \mathrm{MeV} / \mathrm{c}^{2}$. For these reasons, the decay mode is compatible with $\tau \rightarrow \rho(770) v_{\tau}$, whose branching ratio is about $25 \%$.

The second $v_{\tau}$ candidate (Fig. 2, top right) was found in the 2011 data sample [8]. The event has 2 prongs at the primary vertex with the production of a short track with a flight length of $1.54 \mathrm{~mm}$ (associated with a $\tau$ lepton) and a longer track identified as a hadron. The $\tau$ lepton decays into 3 prongs, which are identified as hadrons on the basis of momentum-range consistency. All the kinematical cuts for the selection of $\tau \rightarrow 3 h$ decays are satisfied.

The third $v_{\tau}$ candidate (Fig. 2, bottom right) was observed in the 2012 data sample [9]. The primary vertex is defined by two tracks and a $\gamma$-ray. One of the tracks is identified as being a hadron based on momentum-range selection criteria. The other track is identified as a $\tau$ lepton decaying into a muon; the decay daughter matches with the muon track reconstructed by the electronic detectors, with a measured momentum of $(2.8 \pm 0.2) \mathrm{GeV} / \mathrm{c}$ and a negative charge assessed with a $5.6 \sigma$ significance. The kinematical analysis of the event finally satisfies all the specified criteria for the $\tau \rightarrow \mu$ decay channel.

The fourth $v_{\tau}$ candidate (Fig. 2, bottom left) was found in the 2012 data sample [10]. The primary vertex is defined by four tracks and two $\gamma$-rays. One track is the parent track showing a kink topology with an angle of $(137 \pm 4) \mathrm{mrad}$. The flight length is $(1090 \pm 30) \mu \mathrm{m}$. The kink daughter track has a momentum of $\left(6.0_{-1.2}^{+2.2}\right) \mathrm{GeV} / \mathrm{c}$. Its impact parameter with respect to the primary vertex is $(146 \pm 5) \mu \mathrm{m}$. The daughter track was followed in the downstream bricks till the end of the target. It is exiting the target, stopping in the spectrometer after leaving a signal in three RPC planes. Momentum-range correlation allows to identify it as a hadron.

A fifth $v_{\tau}$ candidate was recently found in 2012 data sample [11] and is shown in Fig. 3 and Fig. 4. The primary vertex consists of the $\tau$ candidate track and a charged particle track which is identified as a hadron from its interaction in the downstream brick. The $\tau$ candidate decays after a flight length of $960 \pm 30 \mu \mathrm{m}$ into one charged daughter which interacts after crossing 22 plates and therefore is identified as a hadron. The difference in angle between the $\tau$ candidate track and the 


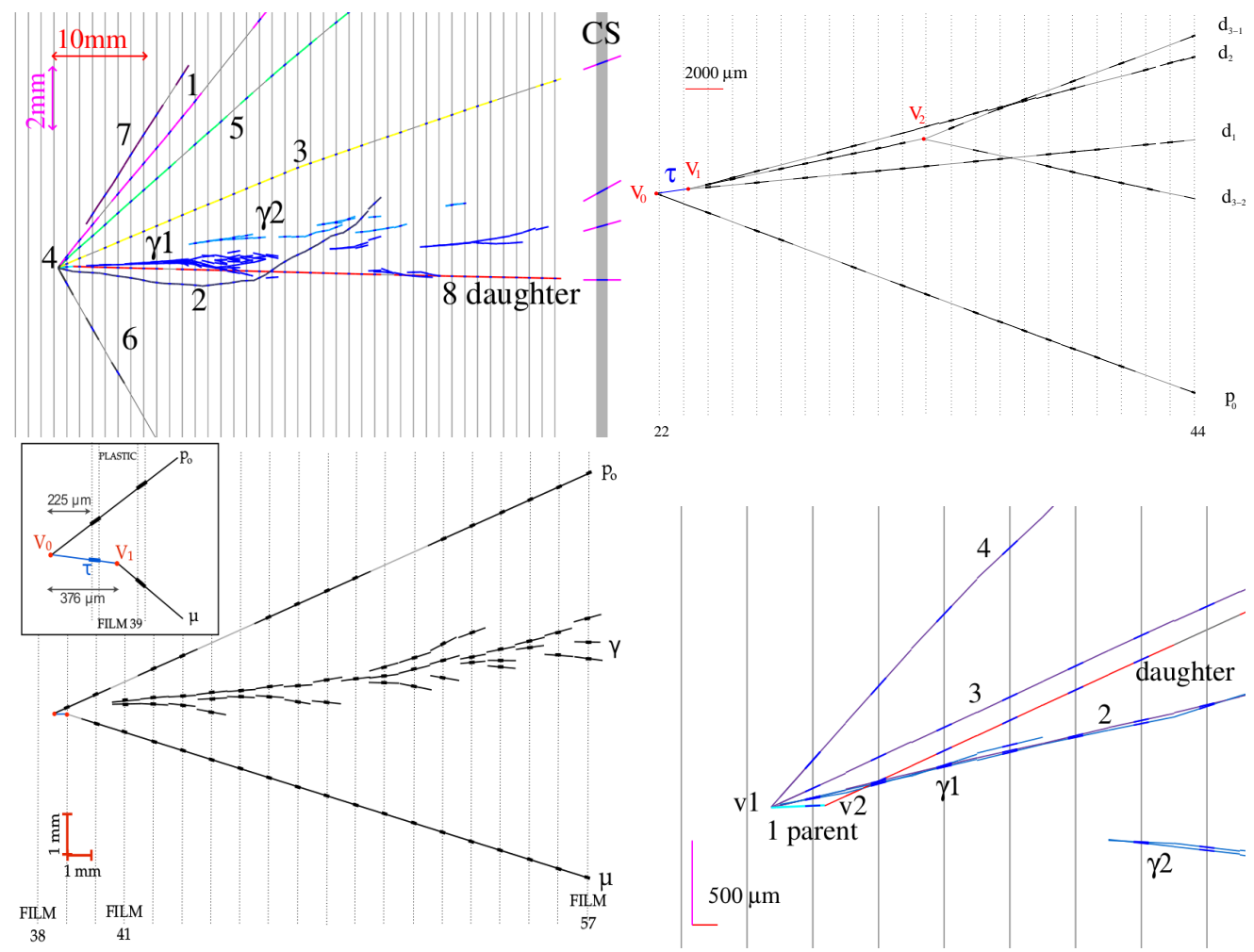

Figure 2: Event displays of the first four observed $v_{\tau}$ candidates (side-views in the brick). Vertical lines indicate the position of the middle-point of emulsion films, numbering them in order of increasing $\mathrm{z}$ from 1 to 57. The pitch is $1.3 \mathrm{~mm}$. The $\tau \rightarrow 1 h$ of [7] (top left), $\tau \rightarrow 3 h$ of [8] (top right), $\tau \rightarrow \mu$ of [9] (bottom left), $\tau \rightarrow 1 h$ of [10] (bottom right) are shown.

daughter particle track, $\theta_{\text {kink }}$, is $90 \pm 2 \mathrm{mrad}$. A search for photon conversions possibly pointing to the primary and secondary vertices was performed. None was found.

In the analysed sample $0.25 \pm 0.05$ background events are expected, coming essentially from charmed events with an undetected primary muon, hadronic re-interactions (for the hadronic decay channels) and large angle muon scattering (for the $\tau \rightarrow \mu$ channel) as shown in Tab. 2. Taking into account the different signal-to-noise ratio for each decay channel, the five observed candidates give a $5.1 \sigma$ significance for the exclusion of the background-only hypothesis in the search for $v_{\mu} \rightarrow v_{\tau}$ oscillations [11].

\begin{tabular}{c|cccc|c|c}
\hline \hline \multirow{2}{*}{ Channel } & \multicolumn{4}{|c|}{ Expected background } & \multirow{2}{*}{ Expected signal } & \multirow{2}{*}{ Observed } \\
\cline { 2 - 6 } & Charm & Had. re-interac. & Large $\mu$-scat. & Total & & \\
\hline$\tau \rightarrow 1 h$ & $0.017 \pm 0.003$ & $0.022 \pm 0.006$ & - & $0.04 \pm 0.01$ & $0.52 \pm 0.10$ & 3 \\
$\tau \rightarrow 3 h$ & $0.17 \pm 0.03$ & $0.003 \pm 0.001$ & - & $0.17 \pm 0.03$ & $0.73 \pm 0.14$ & 1 \\
$\tau \rightarrow \mu$ & $0.004 \pm 0.001$ & - & $0.0002 \pm 0.0001$ & $0.004 \pm 0.001$ & $0.61 \pm 0.12$ & 1 \\
$\tau \rightarrow e$ & $0.03 \pm 0.01$ & - & - & $0.03 \pm 0.01$ & $0.78 \pm 0.16$ & 0 \\
\hline Total & $0.22 \pm 0.04$ & $0.02 \pm 0.01$ & $0.0002 \pm 0.0001$ & $0.25 \pm 0.05$ & $2.64 \pm 0.53$ & 5 \\
\hline \hline
\end{tabular}

Table 2: Expected signal and background events for the analysed data sample. 


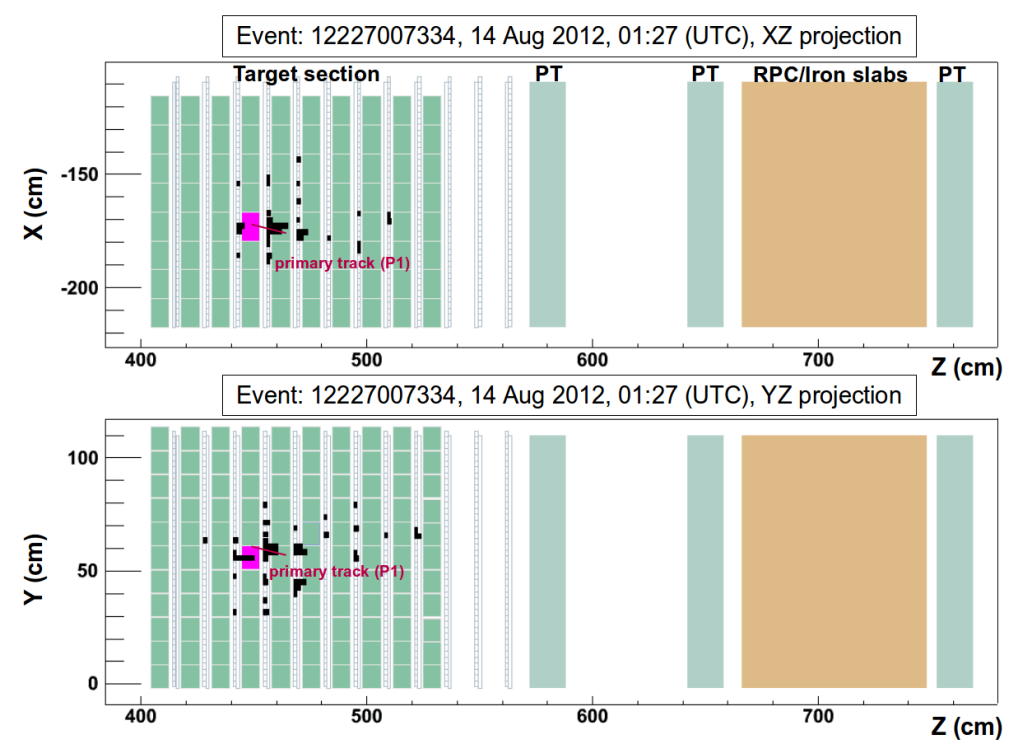

Figure 3: Display of the fifth $v_{\tau}$ candidate event as seen by the electronic detectors. The angle between the neutrino direction and the $z$-axis projected into the $y z$ plane is $58 \mathrm{mrad}$. The brick containing the neutrino interaction is highlighted in magenta. The solid line shows the direction of the primary hadron track (see text) at its most upstream point as reconstructed in the emulsion detectors.

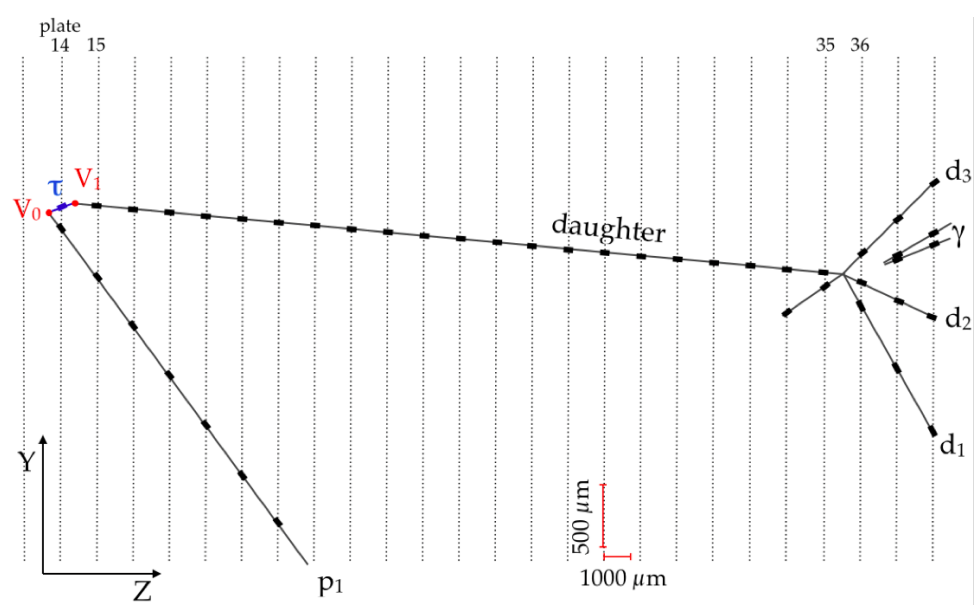

Figure 4: Event display of the fifth $v_{\tau}$ candidate event in the horizontal projection longitudinal to the neutrino direction. The primary and secondary vertices are indicated as " $V_{0}$ " and " $V_{1}$ ", respectively. The black stubs represent the track segments as measured in the films.

\section{Results of the $v_{e}$ searches. Limits on sterile $v$.}

A systematic search for $v_{e}$ events was performed with the 2008-2009 data sample, where 505 $0 \mu$ events were located, corresponding to an integrated intensity of $5.25 \cdot 10^{19}$ p.o.t. [12]. As a result, $19 v_{e}$ candidate events have been observed. This number is compatible with the expected $v_{e}$ from the beam contamination (19.8 \pm 2.8$)$. As a consequence, the current search for the threeflavour neutrino oscillation yields an upper limit $\sin ^{2} 2 \theta_{13}<0.44$ (90\% C.L.). 
The OPERA $v_{e}$ and $v_{\tau}$ results are used to derive limits on the mixing parameters of a massive sterile neutrino [12, 13]. For more details, see L. Stanco, in these proceedings [14].

\section{Measurement of the cosmic muon charge ratio}

The Gran Sasso laboratory is a privileged location to study TeV-scale cosmic rays. A sample of more than 3 million charge-separated atmospheric muon events were reconstructed, among which about 110000 multiple muon bundles. The charge ratio $R_{\mu}=N_{\mu^{+}} / N_{\mu^{-}}$was measured separately for single and for multiple muon events. $R_{\mu}$ depends on the chemical composition and energy spectrum of the primary cosmic rays, on the features of hadronic interactions, and, at high energy, on the prompt component (i.e. muons from charm decays). Hadronic interactions can be studied in the so-called "fragmentation region" in a complementary way with respect to collider experiments. Finally this observable is interesting to validate the calculations of atmospheric neutrino fluxes.

The analysis [15] exploited the inversion of the magnet polarity which was performed in 2012. The combination of the two data sets with opposite magnet polarities allowed minimising systematic uncertainties and reaching an accurate determination:

$$
\begin{aligned}
& R_{\mu}\left(n_{\mu}=1\right)=1.377 \pm 0.006(\text { stat } .)_{-0.001}^{+0.007} \\
& R_{\mu}\left(n_{\mu}>1\right)=1.098 \pm 0.023(\text { stat } .)_{-0.013}^{+0.015}
\end{aligned}
$$

Data were fitted to obtain relevant parameters on the composition of primary cosmic rays and the associated kaon production in the forward fragmentation region. In the surface energy range 1-20 TeV investigated by OPERA, $R_{\mu}$ is well described by a parametric model including only pion and kaon contributions to the muon flux, showing no significant contribution of the prompt component (see Fig. 5). The energy independence supports the validity of Feynman scaling in the fragmentation region up to $200 \mathrm{TeV} /$ nucleon primary energy. It must be noted that this measurement covers a region which had not been previously explored by any other experiment.

\section{Conclusions}

The OPERA experiment has been taking data from 2008 to 2012 , collecting $17.97 \cdot 10^{19}$ p.o.t.; the analysis of a data sample including the first and the second most probable bricks is completed for all runs. In this data sample, five $\tau$ neutrino candidate have been found. Furthermore, a revision of the background estimate in the muonic decay channel has been performed.

Given the low background level and the observed number of $v_{\tau}$ candidate events, the discovery of $v_{\tau}$ appearance in the CNGS neutrino beam is achieved with a significance of $5.1 \sigma$.

The analysis of the collected electron neutrino sample and the analysis of the muon charge ratio in the cosmic ray sample have been also reported.

\section{References}

[1] M. Guler et al. (OPERA Collab.), CERN-SPSC-97-24, 1997.

[2] M. Guler et al. (OPERA Collab.), CERN-SPSC-2001-25, 2001. 


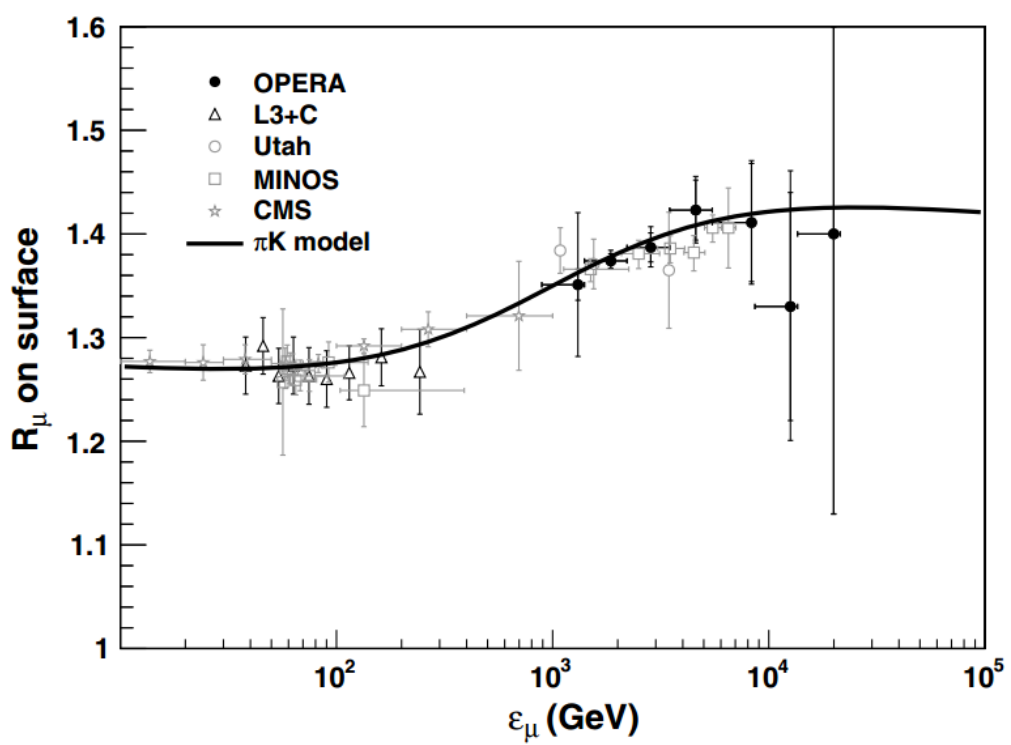

Figure 5: Measurement of the muon charge ratio as a function of the surface energy $E_{\mu}$ (black points). A two-dimensional fit in $\left(E_{\mu}, \cos \theta^{*}\right)$ yields a measurement of the composition parameter $\delta_{0}$ (describing the proton/neutron fraction in cosmic rays nuclei) and of the $Z_{p K^{+}}$moment (related to the associated production of Lambda, $K^{+}$in the forward region). The fit result is shown by the continuous line. From [15].

[3] R. Acquafredda et al. (OPERA Collab.), JINST 4 (2009) P04018.

[4] R. Acquafredda et al. (OPERA Collab.), New J. Phys. 8 (2006) 303.

[5] N. Agafanova et al. (OPERA Collab.), New J. Phys. 13 (2011) 053051.

[6] N. Agafanova et al. (OPERA Collab.), New J. Phys. 14 (2012) 013026.

[7] N. Agafanova et al. (OPERA Collab.), Phys. Lett. B 691 (2010) 138.

[8] N. Agafanova et al. (OPERA Collab.), JHEP 11 (2013) 036.

[9] N. Agafanova et al. (OPERA Collab.), Phys. Rev. D 89 (2014) 051102.

[10] N. Agafanova et al. (OPERA Collab.), Prog. Theor. Exp. Phys. (2014) 101C01.

[11] N. Agafanova et al. (OPERA Collab.), Phys. Rev. Lett. 115 (2015) 12121802.

[12] N. Agafanova et al. (OPERA Collab.) JHEP 07(2013) 004.

[13] N. Agafonova et al. (OPERA Collab.) JHEP 1506 (2015) 069.

[14] L. Stanco, PoS (EPS-HEP2015) 049.

[15] N. Agafonova et al. , Eur. Phys. J. C74 (2014) 72933. 